\title{
Kinetically controlled side-wall-functionalization of carbon nanotubes by nitric acid oxidation
}

\author{
Hao Yu*, Yuguang Jin, Feng Peng*, Hongjuan Wang, Jian Yang
}

School of Chemical and Energy Engineering, South China University of Technology, Guangzhou

$$
\text { 510640, China }
$$

Email addresses: yuhao@ @scut.edu.cn (Hao Yu); cefpeng@scut.edu.cn (Feng Peng)

\section{Supporting Information}

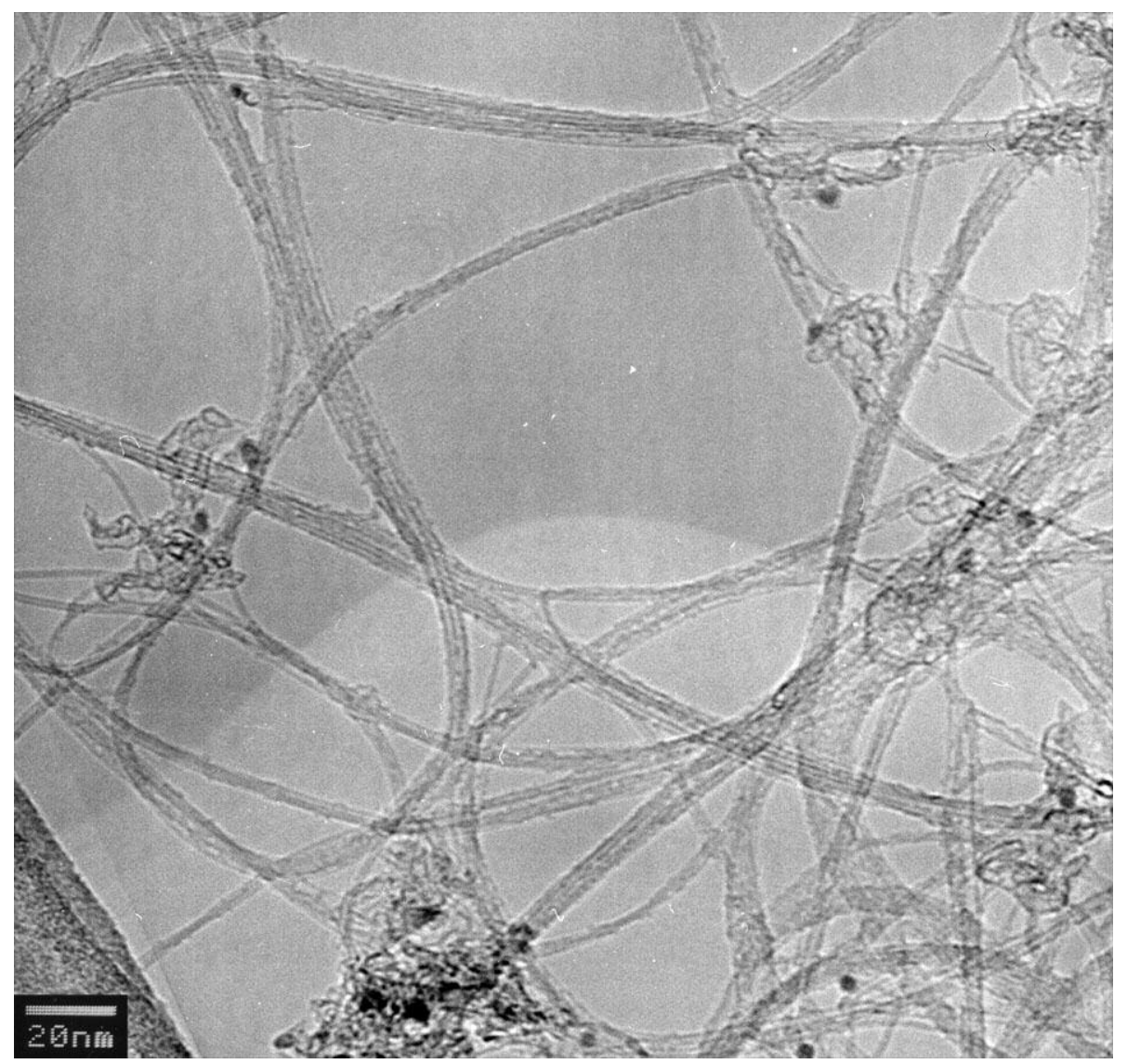

Figure S1 HRTEM image of the pristine SWCNT sample. 


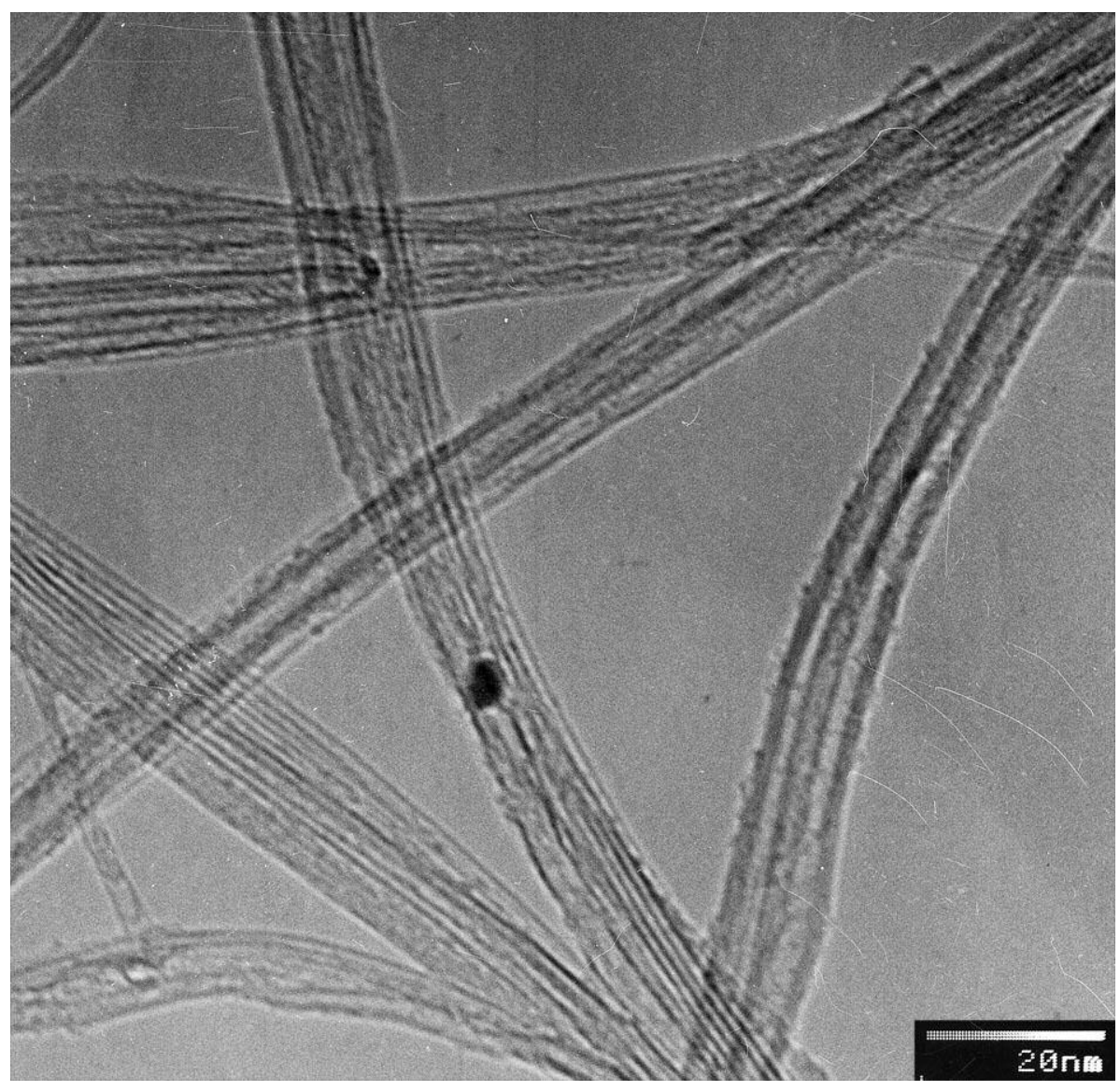

Figure S2 HRTEM image of the pristine FWCNT sample. 


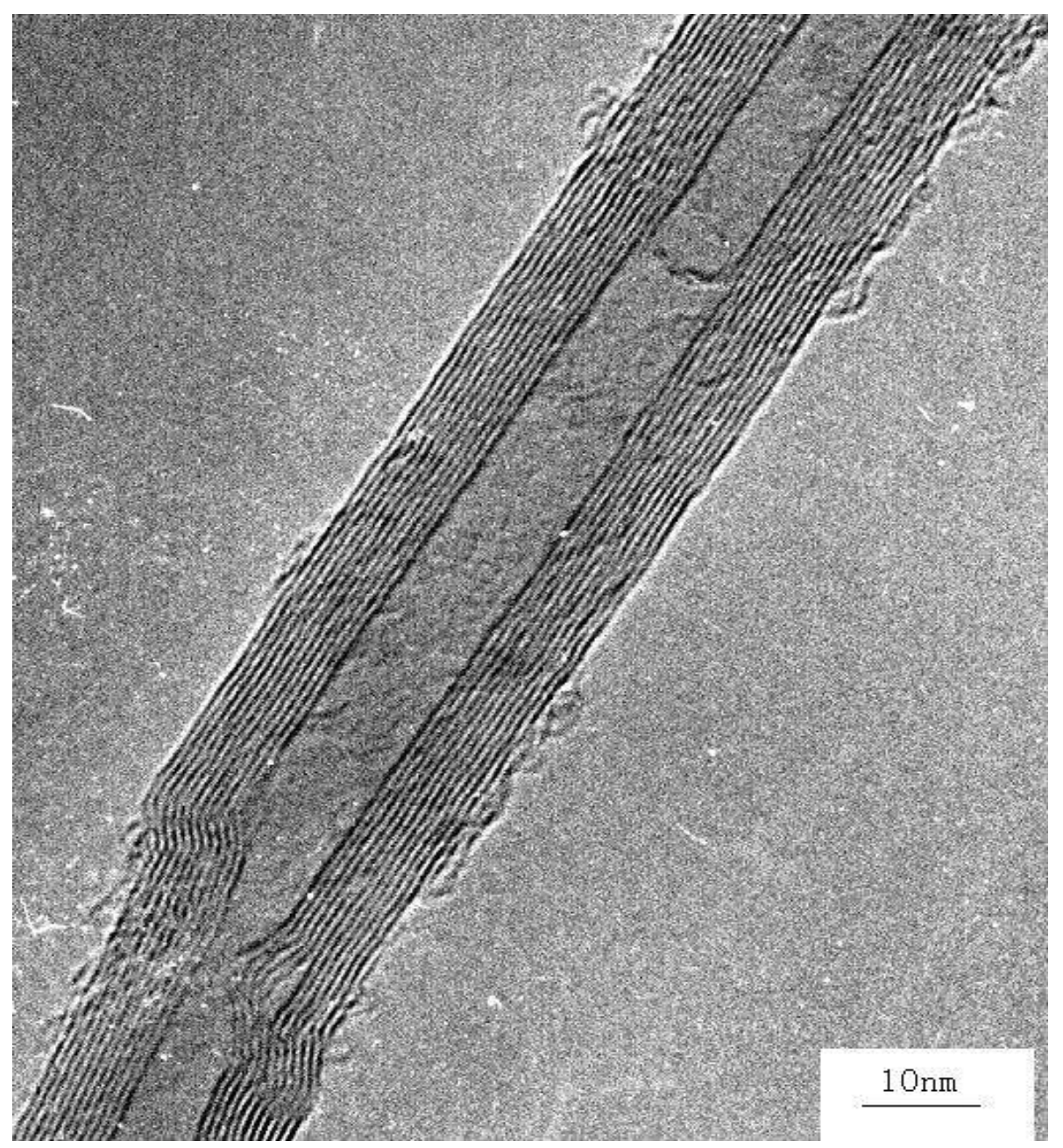

Figure S3 HRTEM image of the high-purity MWCNT sample. 

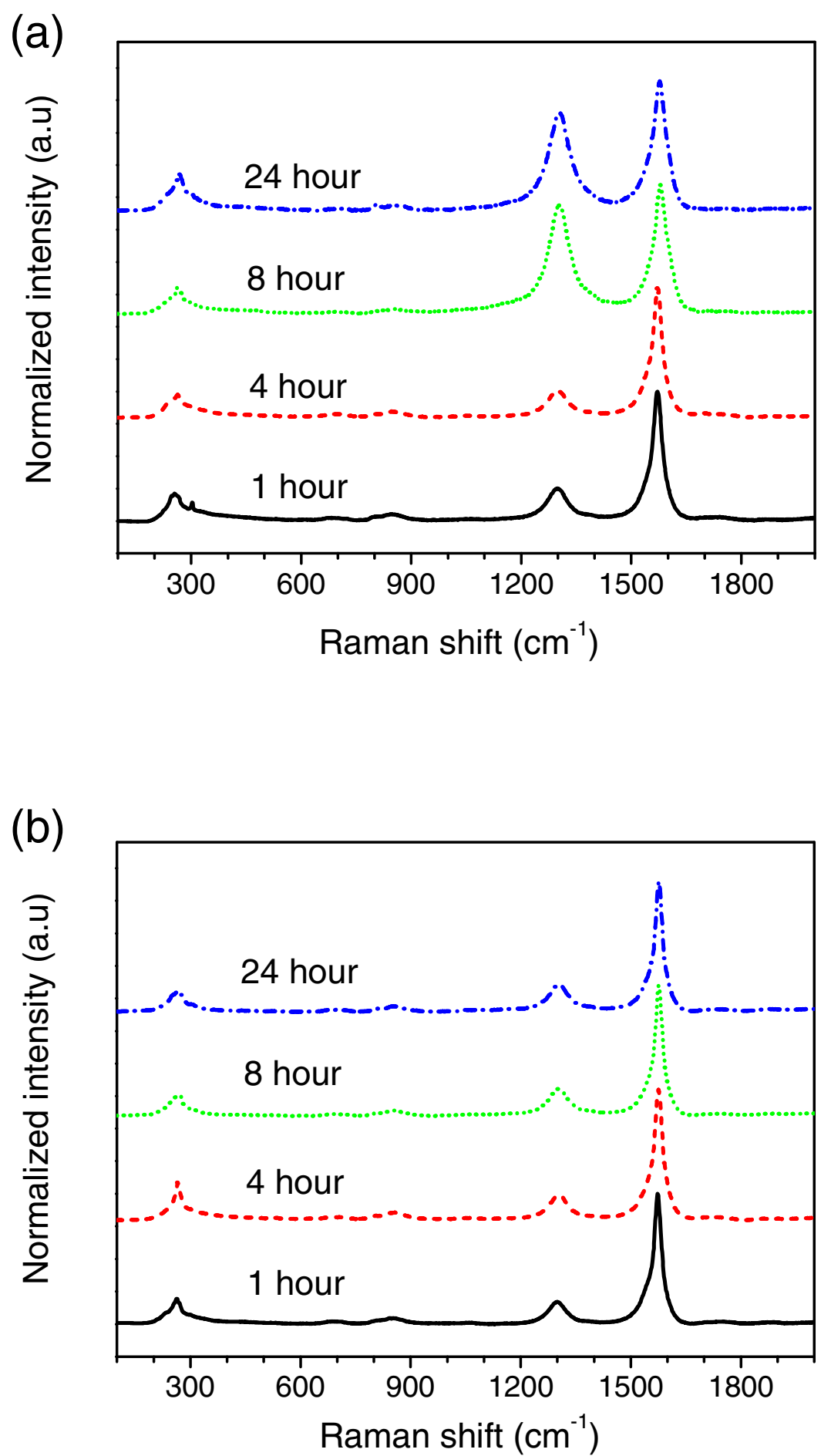

Figure S4 Raman spectra of a) $o$-SWCNTs oxidized by $\mathrm{HNO}_{3}$ for $1 \mathrm{~h}, 4 \mathrm{~h}, 8 \mathrm{~h}$, and $24 \mathrm{~h}$, and (b) corresponding $w$-SWCNTs washed by $\mathrm{NaOH}$ for $48 \mathrm{~h}$. 